\title{
Expression and subcellular distribution of imp13 are regulated in brain development
}

\author{
Pan You • Zi Peng • Yiwei Wang • Tao Tao
}

Received: 30 December 2012 / Accepted: 11 March 2013 /Published online: 20 April 2013 / Editor: T. Okamoto

(C) The Author(s) 2013. This article is published with open access at Springerlink.com

\begin{abstract}
Imp13, a member of importin- $\beta$ superfamily, is found to be one of two bidirectional transport receptors in many nuclear transport activities in mammals. Several cargoes of imp13 have been identified; most of these are essential factors involved in cell cycle and development. The expression and localization of imp13 may influence its cargoes in playing their roles in appropriate time and space. To gain insight into the role of imp13 in brain development, we generated an anti-imp13 polyclonal antibody and investigated the expressions of imp13 in mouse embryonic brains during development, including E13.5, E15.5, E17.5, P0, and adult, at both transcriptional and translational levels. In addition, we performed immunohistochemical analysis and revealed that imp 13 tends to be localized in the cytoplasm at the early stages and relocates into the nucleus at the late stages in neuronal cells of mouse brains. These findings suggested that the expression and localization of imp13 in brain tissues are regulated developmentally, which extends our knowledge of the dynamic presence of imp13. These observations also imply that imp13 contributes to the neural cell-specific cargo trafficking and potentially to other functions during brain development.
\end{abstract}

Keywords Importin13 (impl3) · Imp13 antibody · Brain development $\cdot$ Expression $\cdot$ Localization

P. You $\cdot$ Z. Peng $\cdot$ T. Tao $(\bowtie)$

School of Life Sciences, Xiamen University, Xiamen,

Fujian 361005, China

e-mail: taotao@xmu.edu.cn

Y. Wang

Department of Genetics, Case Western Reserve University, Cleveland, $\mathrm{OH} 44106$, USA

\section{Introduction}

Regulation of nuclear protein transportation through nuclear pore complexes (NPCs) is an essential function in eukaryotic cells. Most macromolecules larger than $40-60 \mathrm{kDa}$ are transported actively through the NPC mediated by transport factors such as members of importin- $\beta$ superfamily (Fabre and Hurt 1994; Davis 1995; Macara 2001; Weis 2002). Importin13 (imp13, lgl2, Kap13, or IPO13), a member of the importin- $\beta$ superfamily, is a nucleus-cytoplasm bidirectional transporter that plays roles in cell differentiation, embryonic development, and physiologic functions of several organs, including lung, brain, and heart (Zhang et al. 2000; Su et al. 2002; Tao et al. 2004; Tao et al. 2006; Lin et al. 2009). Imp13 has been documented to be involved in nuclear import or export of several nuclear proteins which include eIF1A, Mago-Y14 and Ubc9 (Mingot et al. 2001), Pax6 (Ploski et al. 2004), NF-YB/NF-YC dimer (Kahle et al. 2005), the gulcocorticoid receptor (GR) (Tao et al. 2006), myopodin (Liang et al. 2008), and Arx (Shoubridge et al. 2007; Lin et al. 2009). Some of these substrates are important in embryonic neural development. For example, PAX6 is a master control gene for eye morphogenesis (Gehring 1996) and $A R X$ is necessary for the development of forebrain (Lin et al. 2009). Imp13 affects neurotransmitter release at the Drosophila neuromuscular junction (Giagtzoglou et al. 2009). Imp13 is exclusively expressed in the basal cells of human limbal epithelia and regulates differentiation and proliferation of corneal epithelial progenitor cells (Wang et al. 2009). These findings imply that imp13 may play essential roles in neural development.

Expression and localization of nucleocytoplasmic transporters have drawn a lot of interest recently. Importin proteins show regulated expression patterns in cells and tissues 
at both RNA and protein levels, indicating that nuclear import of the cargo proteins depends on the presence of different amounts of importins (Jans et al. 2000; Itman et al. 2009; Yasuhara et al. 2009). Because of the intrinsic selectivity importin proteins display for different cargoes, their differential expression patterns during development and differentiation are suggested to be a potential regulatory mechanism (Yamaguchi et al. 2006; Terry et al. 2007; Okada et al. 2008; Yasuhara et al. 2009; Whiley et al. 2012; Yasuda et al. 2012). Several lines of evidence show regulated importin expression during embryonic development (Kamei et al. 1999; Hogarth et al. 2006; Loveland et al. 2006; Hogarth et al. 2007; Nakamura et al. 2007; Quan et al. 2008). However, neither expression nor subcellular localization of imp13 in brain development has been closely examined. In this study, we generated a rabbit anti-imp13 antibody to analyze both expression and localization of endogenous imp13 in embryonic mouse brain tissues. Our results revealed that both expression and subcellular localization of endogenous imp13 are dynamically changed in some tissues of embryonic mouse brains, suggesting a significance of spatial-temporal regulation of imp13 in mouse brain development.

\section{Materials and Methods}

Preparation of a rabbit anti-imp13 antibody. To express GST-tagged imp13 protein, cells of Escherichia coli BL21 carrying pGEX-4T-2-imp13 (Tao et al. 2004) were grown overnight on LB-Amp plates and were used to inoculate $1 \mathrm{~L}$ of prewarmed LB containing $100 \mu \mathrm{g} / \mathrm{mL}$ ampicillin. The cell cultures were incubated at $37^{\circ} \mathrm{C}$ with vigorous shaking. At an optical density of 0.7 at $600 \mathrm{~nm}$, the cultures were rapidly chilled to $20^{\circ} \mathrm{C}$ and $0.1 \mathrm{mM}$ IPTG was added. Cells were incubated for another $6 \mathrm{~h}$ and cells were harvested by centrifugation. Cells were lysed and GST-tagged imp13 proteins were purified using Glutathione Sepharose 4B beads.

For antibody production, New Zealand white rabbits were immunized subcutaneously using $0.5 \mathrm{mg}$ GST-tagged imp13 proteins per kilogram body weight after emulsified to Freund's complete adjuvant. Two weeks after primary injection, booster injections were made using $0.5 \mathrm{mg}$ fusion protein per kilogram body weight with Freund's incomplete adjuvant. Twelve days after monthly booster injections, antisera were collected from an artery in the ear. Finally, the purified rabbit anti-imp13 antibody was prepared after absorbing GST antibody with GST beads.

Animals and tissues. All animal experimental procedures were conducted in accordance with the guide for the care and use of laboratory animals, provided by the Xiamen University Laboratory Animal Center, Xiamen, China.
C57/BL-6 mouse embryos at different developmental stages were collected (three mice per group) for real-time RT-PCR, Western blot, and immunohistochemical analysis. Brains of E11.5, E13.5, E15.5, and E17.5 were carefully dissected out from the skulls and placed into ice-cold phosphate-buffered saline (PBS); for P0 and adult mice, after a deep anesthesia, the animals were perfused with cold $4 \%$ paraformaldehyde transcardially using a peristaltic perfusion connected with an 18 -gauge needle through the left ventricle and by clamping the descending aorta. Then, after perfusion, the animals were decapitated, the skulls were taken off, and the brains were carefully removed. For immunostaining, all samples were dehydrated and embedded in paraffin and cut into $4-\mu \mathrm{m}$ thick sections with a diamond knife with an ultra-microtome (Leica RM2255, Nussloch, Germany).

Analysis of real-time RT-PCR. Total RNAs were isolated with RNAfast 1000 (Feijie, China) and all primers and probes were ordered from Applied Biosystems (Foster, CA, USA). The sequence of the primers and probes used for TaqMan RT-PCR amplification are as follows: a forward primer (5'-ACAGCCCCAAAATGGTTAAGGTT-3'), a reverse primer (5'-CCAACACTT CGAGAGGTCCTT-3'), and the TaqMan MGB probe (FAM 5'-CCAACACTTCGAG AGGTCCTT-3' NFQ) were used for amplifying a 61-bp fragment of the Hprt1 cDNA as internal control. A forward primer (5'-GGCTCCAAAATTGTGCTGACT-3'), a reverse primer (5'-GCATCATGCTGAGAG CTAGTGA-3'), and a TaqMan MGB probe (FAM 5'-CCAGCGCCACGCACAG$3^{\prime}$ NFQ) were used to amplify a 64-bp fragment of IPO13 cDNA. Analysis of real-time RT-PCR was carried out in a total volume of $20 \mu \mathrm{L}$. The thermal cycle conditions were as follows: $1 \times 2 \min 50^{\circ} \mathrm{C}, 1 \times 10 \min 95^{\circ} \mathrm{C}$, followed by 40 cycles of reaction at $95^{\circ} \mathrm{C}$ for $15 \mathrm{~s}$ and combined annealing/extension step at $60^{\circ} \mathrm{C}$ for $1 \mathrm{~min}$. Expression of IPO13 was normalized to HPRT using the $2^{-\Delta \Delta \mathrm{Ct}}$ method as described (Tichopad et al. 2003; Kane et al. 2005).

Western blot analysis. Tissues were pulverized with a mortar and pestle on ice and homogenized in the lysis buffer (50 mM HEPES-NaOH, pH 7.5, $100 \mathrm{mM} \mathrm{NaCl}, 0.5 \%$ NP40, 2.5 mM EDTA, 10\% glycerol, 1 mM DTT, 1 mM PMSF, and $1 \%$ proteinase inhibitor cocktail). The homogenates were centrifuged for $20 \mathrm{~min}$ at $12,000 \times \mathrm{g}$ at $4^{\circ} \mathrm{C}$. Protein concentrations in the supernatants were measured with a spectrophotometer (Bio-Rad SmartSpec 3000, El Cajon, CA, USA). Protein extracts ( $60 \mu \mathrm{g}$ per sample) were denatured by boiling in $2 \times$ loading buffer $(0.25 \mathrm{M}$ Tris- $\mathrm{HCl}$, $20 \%$ glycerol, $4 \%$ SDS, $0.005 \%$ bromoethanol blue, and $5 \%$ $\beta$-mercaptoethanol) for $5 \mathrm{~min}$, separated by $10 \%$ SDSPAGE, and transferred to a PVDF membrane. The membrane was placed in blocking solution (5\% non-fat dry milk in TBST) for $1 \mathrm{~h}$ at room temperature and then incubated 

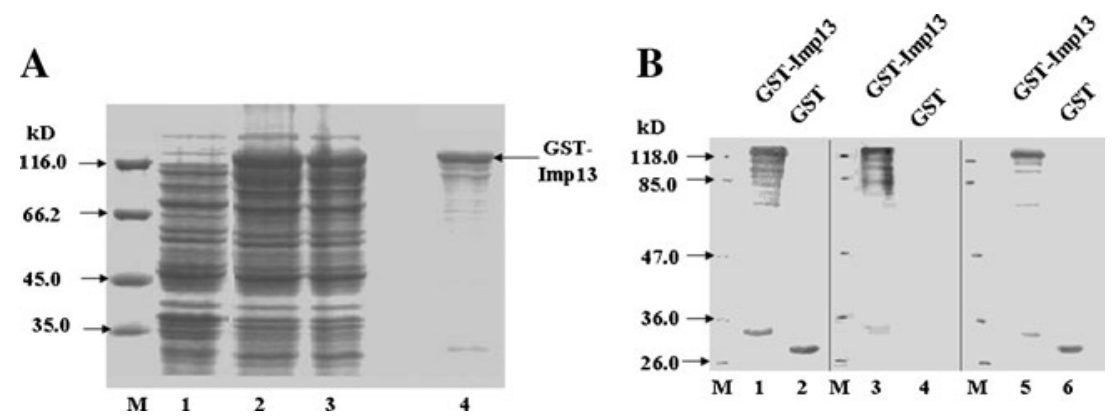

Figure 1. Preparation of a rabbit anti-imp13 antibody. (A) SDSPAGE of GST-tagged imp13 proteins. Lane 1 whole cell lysates before IPTG induction. Lane 2 whole cell lysates after IPTG induction. Lane 3 the supernatant of cell lysates after IPTG induction. Lane 4 purified GST-tagged imp13 using Glutathione Sepharose 4B beads. (B) Western blot analysis for the retained GST antibody in the anti-sera. GSTtagged imp13 and GST proteins were separated by $10 \%$ SDS-PAGE

overnight at $4^{\circ} \mathrm{C}$ with either the rabbit anti-imp 13 polyclonal antibody at 1:1,000 dilution or a mouse anti-tubulin monoclonal antibody at 1:1,000 dilution (\#MAB5564, Chemicon,

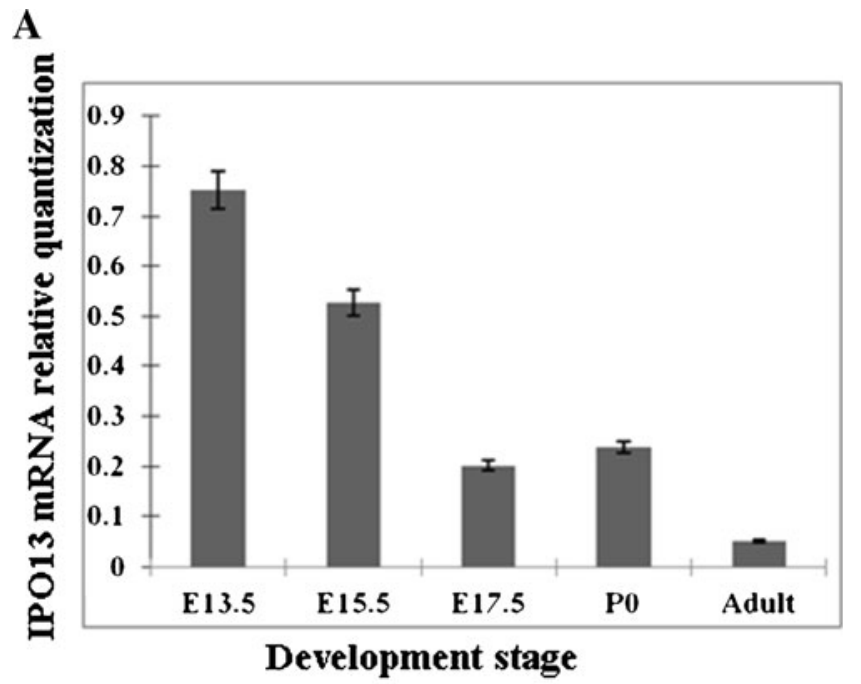

$\mathbf{B}$

\section{$\operatorname{Imp} 13$}

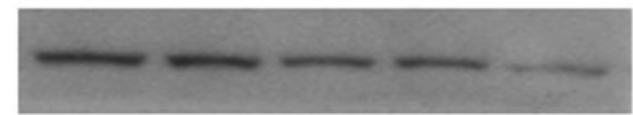

Tubulin

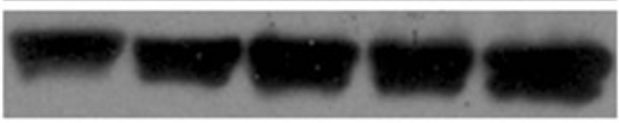

\section{E13.5 E15.5 E17.5 P0 Adult}

Figure 2. Expressions of imp13 in fetal brains at the indicated stages. $A$ Expressions of IPO13 in the brains at the indicated stages were analyzed with real-time RT-PCR analyses. $B$ Expressions of imp13 protein in mouse fetal brain at the indicated stages were analyzed with Western blot analyses. Relative quantification real-time RT-PCR analyses were normalized to Hprt loading controls. Error bars represent the standard error of the mean (SEM); Western blot analyses were normalized against tubulin loading controls. and proteins were transferred to a nitrocellulose membrane. Lanes 1-4 blots were probed with anti-serum prepared primary antibody (1:2,000), followed by goat-anti rabbit IgG/HRP secondary antibody. And retained GST antibody was detected in the anti-serum before treatment (lanes 1 and 2). No GST antibody was detected in the antisera after treatment with GST beads (lanes 3 and 4). Lanes 5-6 blots were probed with anti-GST primary antibody $(1: 1,000)$ as a control.

Temecula, CA, USA). The membrane was rinsed three times, for a total of $30 \mathrm{~min}$ in TBST, followed by incubation with horseradish peroxidase-conjugated anti-rabbit or anti-mouse IgG antibodies. The membrane was rinsed three times again for 30 min total in TBST and developed with ECL plus Western Blotting Detection Reagents (GE Healthcare, Piscataway, New Jersey, USA).

Immunostaining. Immunohistochemical analysis was performed using the rabbit anti-imp13 polyclonal antibody, a rabbit anti-imp9 polyclonal antibody (\#ab52605; Abcam, UK), and a rabbit anti-imp $\beta 1$ polyclonal antibody (\#ab2811; Abcam). Four-micrometer-thick serial sections were mounted on glass slides coated by 3 -aminopropyltriethoxysilane, and the slides were dried overnight at $37^{\circ} \mathrm{C}$ followed by incubating for $30 \mathrm{~min}$ at $64^{\circ} \mathrm{C}$. The sections were deparaffinized in two changes of xylene and rehydrated in a series of descending concentrations of ethanol. Antigen retrieval was performed by microwave heating the slides in $10 \mathrm{mM}$ citrate buffer for $20 \mathrm{~min}$. The sections were then incubated with primary antibodies at $4^{\circ} \mathrm{C}$ overnight. After washing with PBS solution three times for $5 \mathrm{~min}$, the sections were incubated with horseradish peroxidase-conjugated anti mouse/rabbit immunoglobulin $\mathrm{G}$ (secondary antibodies) for $30 \mathrm{~min}$ at room temperature. The immune complex was visualized by incubation with diaminobenzidine (DAB Kit; Maixin Biological, Fuzhou, China) for $5 \mathrm{~min}$. The sections were then lightly counterstained with hematoxylin, dehydrated, and mounted. For negative controls, the Tris-buffered saline (TBS) solution was used instead of the primary antibody.

\section{Results}

Preparation of rabbit anti-imp13 antibody. GST-tagged full-length imp 13 protein was expressed and partially purified 
in bacterial cells (Fig. 1A, lanes 2, 3, and 4). Because the rabbits were immunized against a GST-tagged imp13, their serum should contain antibodies against imp 13 and antibodies against the GST tag as well (Fig. $1 B$, lanes 1 and 2). By incubation of the serum with GST immobilized Glutathione Sepharose 4B beads, the anti-GST antibodies in the serum were removed and the treated serum does not recognize the GST tag but is able to detect imp13 (Fig. 1B, lanes 3 and 4). Therefore, we generated an anti-imp13 antibody which was used in the present study.

Expression of imp13 in the brain is temporal-specific during development. The expression of imp13 has been suggested to be regulated in multiple fetal tissues (Quan et al. 2008). Since imp13 transports several transcription factors which are essential in brain development (Ploski et al. 2004; Lin et al. 2009), it is of interest to examine the expression of imp13 in mouse brain development. To this end, we carried out two parallel experiments. First, we applied the real-time RT-PCR analysis to quantify its mRNA levels of IPO13 from fetal brain tissues of mice. Total RNAs were isolated from the brain at E13.5, E15.5, and E17.5 embryos as well as at P0 and of adult mice. The level of IPO13 mRNA was normalized to HPRT internal control and presented in Fig. 2A. Interestingly, IPO13 expression level in fetal brain tissues was found to be the highest at E13.5 and then gradually decreased. By contrast, the expression of IPO13 from adult mouse brain tissues was shown to be the lowest level among the time point examined. Meanwhile, Western blot analyses further confirmed the temporal-specific expression pattern of imp13 (Fig. 2B).
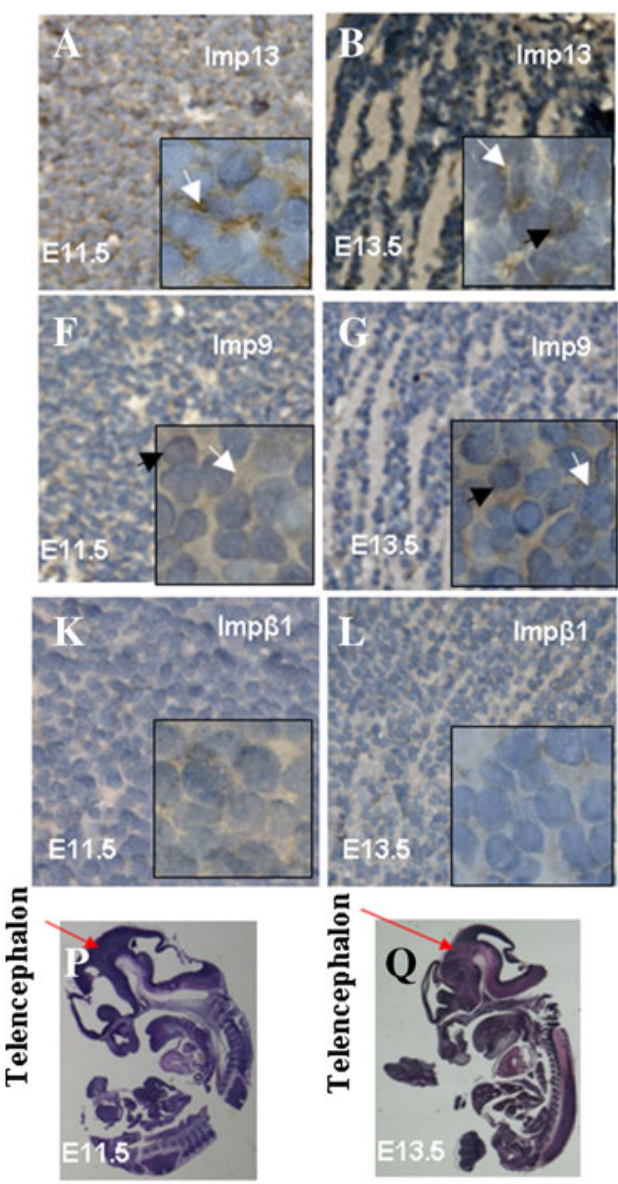
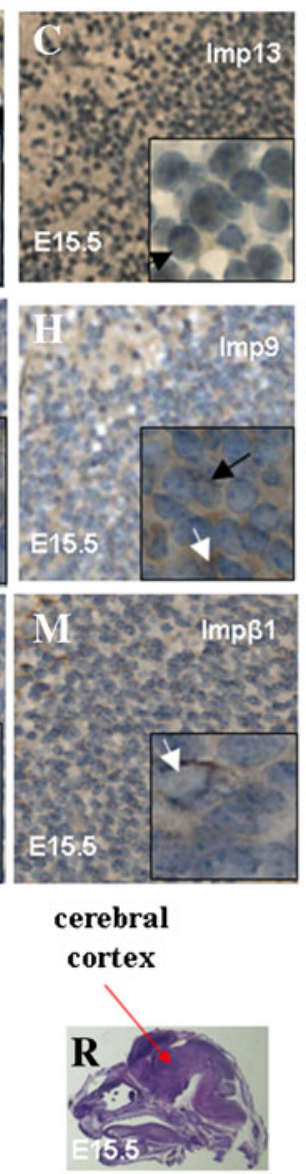
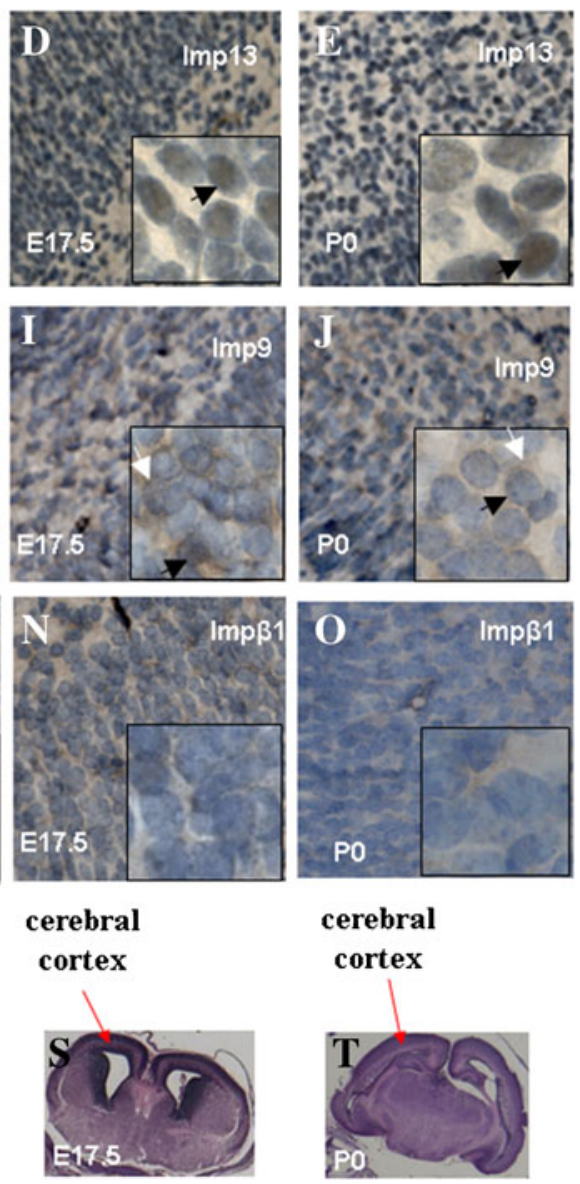

cerebral

cortex

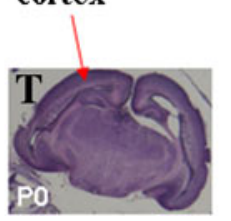

Figure 3. Cellular localization of imp13 $(A-E)$, imp $9(F-J)$, and imp $\beta 1(K-O)$ in fetal and nascent brain. $A-O$, Immunohistochemical staining of sagittal sections (E11.5-E15.5) or coronal section (E17.5 and $\mathrm{P} 0$ ) of the brains at the indicated stages was employed to assess cellular localization of imp13, imp9, or imp $\beta 1$. Black arrows indicate nuclear localizations and white arrows indicate cytosol localizations. $\operatorname{Imp} \beta 1$ is expressed lowly during brain development $(K-O) . P-T \mathrm{H} \& \mathrm{E}$ staining of sagittal sections (E11.5-E15.5) or coronal section (E17.5 and $\mathrm{P} 0$ ) of whole mouse embryos or brains at the indicated stages. Red arrows indicate fetal and nascent mouse telencephalon $(P, Q)$ or cerebral cortex $(R-T) . A, F, K: \mathrm{E} 11.5 ; B, G, L: \mathrm{E} 13.5 ; C, H, M$ : $\mathrm{E} 15.5 ; D, I, N$ : E17.5; $E, J, O$ : P0 (original magnification $\times 400$ ). The inset is the high magnification view (original magnification $\times 1,000$ ). 
In order to localize imp13 in developing brains, we then employed the immunohistochemical staining (IHC) method. Distribution of endogenous imp 9 and imp $\beta 1$, two other members of the importin- $\beta$ super family, was used as control. In the tissue of telencephalon, endogenous imp13 was mainly localized in the cytoplasm of at E11.5 (Fig. $3 A$ ). At E13.5, imp13 is in both the cytoplasm and nucleus (Fig. $3 B$ ). By contrast, at later stages from E15.5 to P0, imp13 is mainly in the nucleus and only a weak staining of imp13 can be observed in the cytoplasm (Fig. 3C-E). Endogenous imp9 resides mainly in the cytoplasm of telencephalon in all stages (Fig. $3 F-J$ ). A similar distribution pattern of imp13 was also observed in cerebellar primordium (Fig. $4 A-C$ ), striatum (Fig. 5A, $B$ ), and external capsule (Fig. $5 I, J$ ). In the hippocampus, however, imp13 was found to be in both the cytoplasm and the nucleus from the early to late stage (Fig. $5 Q, R$ ), which was similar to that of imp9 (Fig. 5S, T) and imp $\beta 1$ (Fig. 5U, V). This may suggest the existence of different neural progenitor cells in the hippocampus area (Hildrestrand et al. 2009). Only a weak staining of imp $\beta 1$ could be observed in the mouse developing brain (Fig. $3 K-O$; Fig. $4 G-I$; Fig. $5 E-F, M-N, U-V$ ).

\section{Discussion}

In this work, an antibody against full-length imp13 was generated and the expression and subcellular distribution of endogenous imp13 during the development of embryonic mouse brain were systematically examined. Both the expression and the subcellular distribution of imp13 in fetal brain are found to be regulated: at early developmental stages, its expression level is higher than that at later stages. Imp13 is mainly in the cytoplasm of telencephalon at early developmental stages, but it is concentrated in the nucleus of telencephalon when mice were born. Since imp 13 is one of importin- $\beta$ superfamily which plays crucial roles in nucleocytoplasmic transport, our results suggest that its function is probably regulated spatialtemporally in brain development.

The nucleocytoplasmic transport has been suggested to be highly regulated at different levels such as cargo, importin- $\beta$ s, and NPCs (Major et al. 2011). Even subtle changes of them may result in significant impact on biological processes and dramatic influences on cell signaling. Imp13-mediated nuclear import of transcription factors in response to regulatory signaling governs organogenesis
Figure 4. Cellular localization of imp13 $(A-C)$, imp9 $(D-F)$, and $\operatorname{imp} \beta 1(G-I)$ in fetal mouse cerebellar primordium or cerebellum at differential stages. Black arrows indicate nuclear localizations and white arrows indicate cytosol localizations. $\operatorname{Imp} \beta 1$ is expressed lowly during cerebellum development $(G-I)$. Red arrows indicate fetal mouse cerebellar primordium $(J, K)$ or cerebellum (L). A, D, G: E11.5; $B, E, H$ : E13.5; $C, F, I: \mathrm{E} 15.5$ (original magnification $\times 400$ ). The inset is the high magnification view (original magnification $\times 1,000$ ).
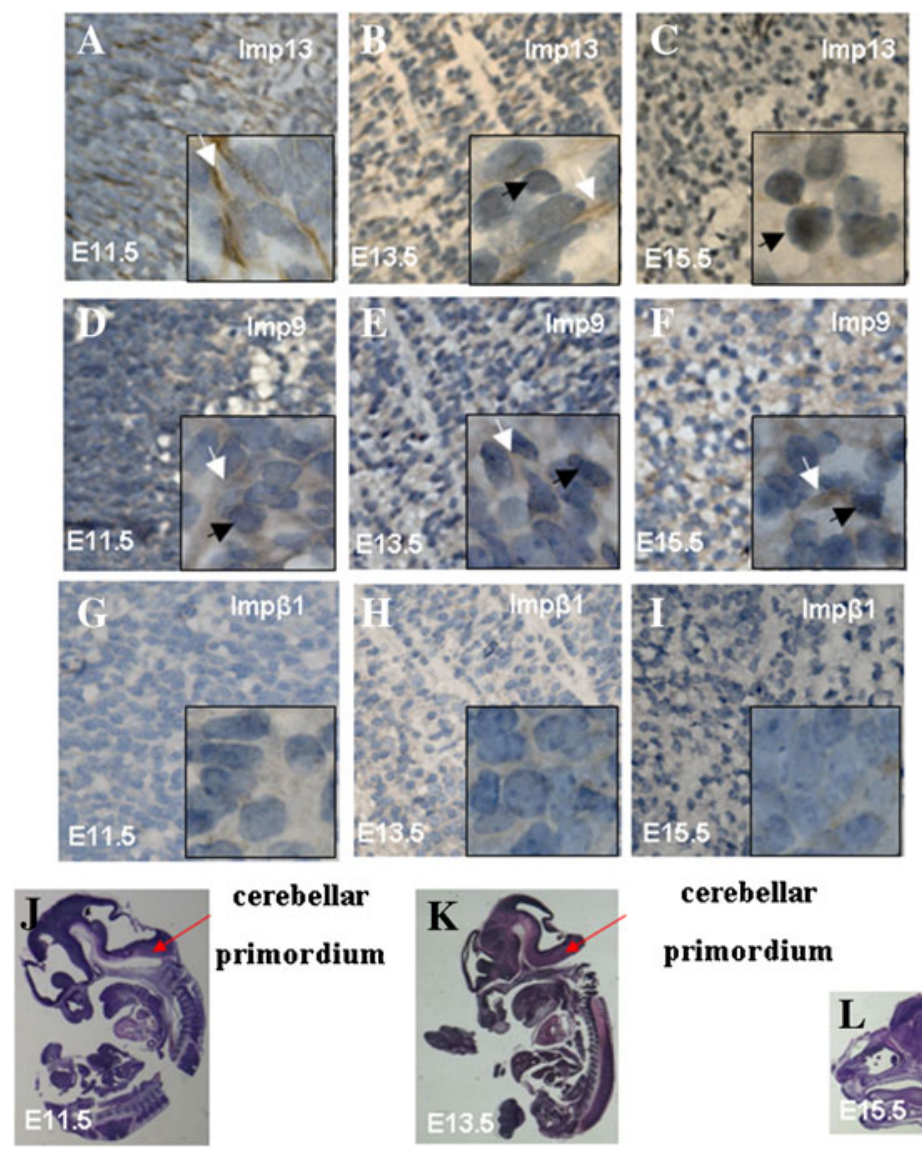

cerebellar

primordium

cerebellum

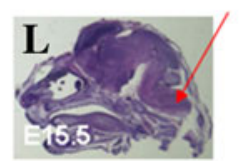



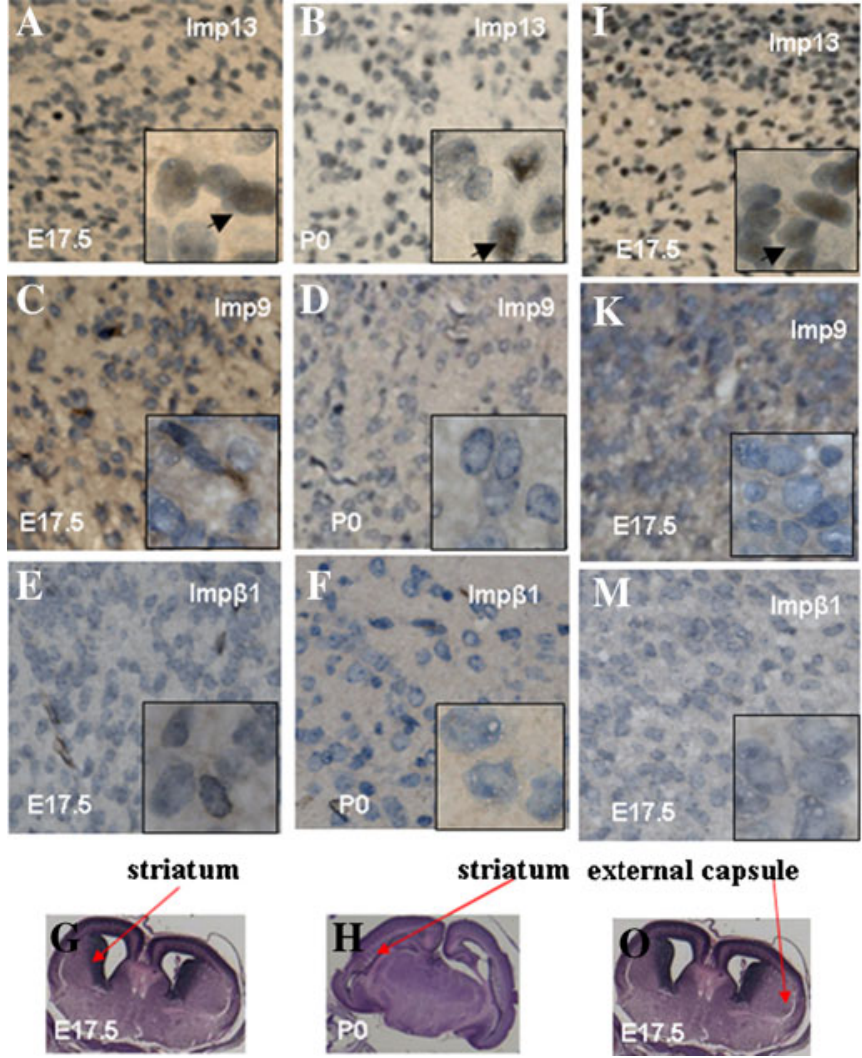

triatum external capsule

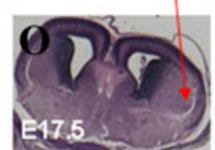

Figure 5. Cellular localization of imp13 $(A, B, I, J, Q, R)$, imp9 $(C, D, K, L, S, T)$, and impß1 $(E, F, M, N, U, V)$ in fetal mouse and neonatal (P0) mouse's striatum, external capsule, and hippocampus at differential stages. Black arrows indicate nuclear localizations and white arrows indicate cytosol localizations; imp9 and $\operatorname{imp} \beta 1$ are expressed lowly in the striatum $(C, D, E, F)$ and

during embryonic development. We have reported previously that the imp13 primarily is localized in the nucleus in fetal lung and that imp13 enters the nucleus much more rapidly at E18 than at E21 (Tao et al. 2004). Total nuclear recovery of imp13 was significantly different at these two time points. In this study, different subcellular distribution patterns of imp13 are found during the development of fetal brain comparing with imp $\beta 1$ and imp9, which may indicate its distinct roles in this process. It is also possible that nuclear localization of imp13 may reflect selective nuclear retention to reduce its nucleocytoplasmic transport activities at late brain development.

Regulated synthesis of specific importin proteins has been shown to mediate cell fate outcomes in embryonic stem cell systems (Perez-Terzic et al. 2007; Yasuhara et al. 2007; Young et al. 2011). Expression of imp13 shows a higher level in early developmental brain (Fig. 2A, B), suggesting that there might be a heavier nucleocytoplasmic
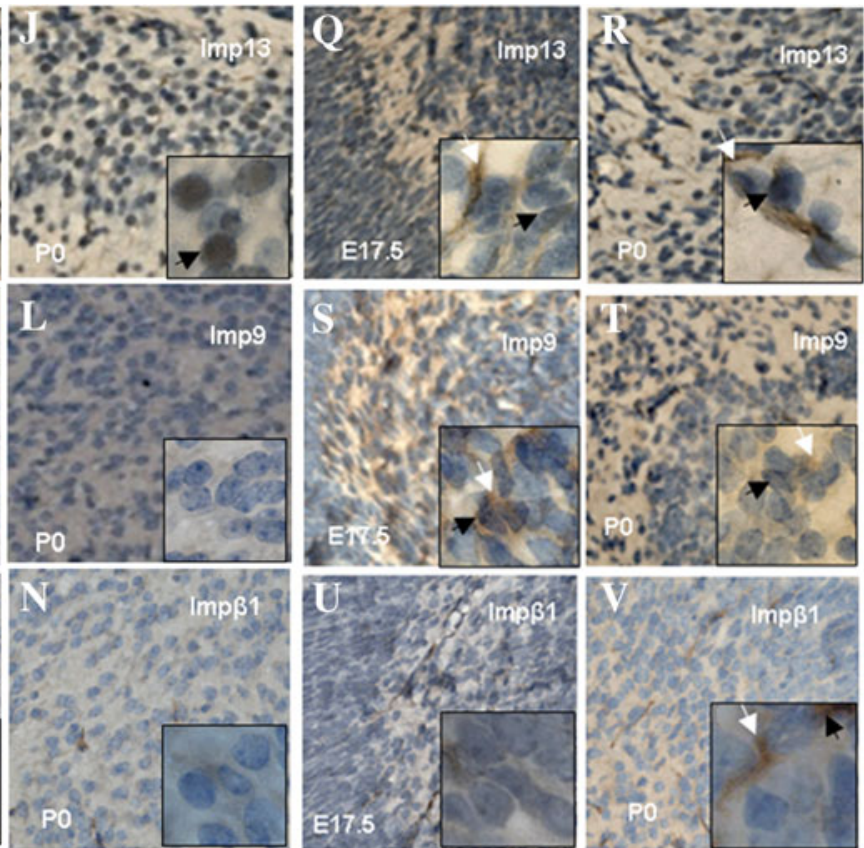

external capsule
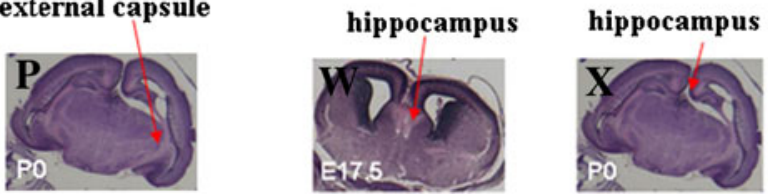

external capsule $(K, L, M, N)$. Red arrows indicate fetal mouse (E17.5) and neonatal (P0) mouse's striatum $(G, H)$, external capsule $(O, P)$, and hippocampus $(W, X) . A, C, E, I, K, M, Q$, $S, U:$ E17.5; $B, D, F, J, L, N, P, T, V:$ P0 (original magnification $\times 400$ ). The inset is the high magnification view (original magnification $\times 1,000$ )

transport of cargoes by imp 13 when mouse neurogenesis starts (Hartfuss et al. 2001; Noctor et al. 2002; Gotz and Huttner 2005).

Imp13 is one of two members in importin- $\beta$ superfamily which can transport cargoes in and out of the nucleus (Mingot et al. 2001). It is not clear when imp13 only/mainly imports or exports cargoes. It is possible that imp 13 mainly functions in importing transcription factors such as Pax6 or Arx at early developmental stages but functions mainly in exporting cargoes such as eIF1A at late developmental stages in this case. Mechanisms in cells to regulate the functional transition of imp13 from import to export or vice versa could be related to unknown factors in brain development.

In summary, subcellular localization of endogenous imp13 is shown for the first time to be regulated throughout fetal brain development, suggesting a potential for neural cell-specific nucleocytoplasmic transport mechanisms to govern brain development. 
Acknowledgments This work was supported by a grant (\#2012CB966602) from the Ministry of Science and Technology, China and a grant (\#30971669) from the National Science Foundation of China to Dr. Tao Tao.

Conflict of interest The authors have declared no conflicts of interest.

Open Access This article is distributed under the terms of the Creative Commons Attribution License which permits any use, distribution, and reproduction in any medium, provided the original author(s) and the source are credited.

\section{References}

Davis L. I. The nuclear pore complex. Annu. Rev. Biochem. 64: 865896; 1995

Fabre E.; Hurt E. C. Nuclear transport. Curr. Opin. Cell Biol. 6: 335342; 1994

Gehring W. J. The master control gene for morphogenesis and evolution of the eye. Genes Cells 1: 11-15; 1996.

Giagtzoglou N.; Lin Y. Q.; Haueter C.; Bellen H. J. Importin13 regulates neurotransmitter release at the Drosophila neuromuscular junction. J. Neurosci. 29(17): 5628-5639; 2009.

Gotz M.; Huttner W. B. The cell biology of neurogenesis. Nat. Rev. Mol. Cell Biol. 6(10): 777-788; 2005.

Hartfuss E.; Galli R.; Heins N.; Gotz M. Characterization of CNS precursor subtypes and radial glia. Dev. Biol. 229(1): 15-30; 2001.

Hildrestrand G. A.; Neurauter C. G.; Diep D. B.; Castellanos C. G.; Krauss S.; Bjoras M.; Luna L. Expression patterns of Neil3 during embryonic brain development and neoplasia. BMC Neurosci. 10: 45; 2009.

Hogarth C. A.; Calanni S.; Jans D. A.; Loveland K. L. Importin alpha mRNAs have distinct expression profiles during spermatogenesis. Dev. Dyn. 235(1): 253-262; 2006.

Hogarth C. A.; Jans D. A.; Loveland K. L. Subcellular distribution of importins correlates with germ cell maturation. Dev. Dyn. 236(8): 2311-2320; 2007

Itman C.; Miyamoto Y.; Young J.; Jans D. A.; Loveland K. L. Nucleocytoplasmic transport as a driver of mammalian gametogenesis. Semin. Cell Dev. Biol. 20: 607-619; 2009.

Jans D. A.; Xiao C. Y.; Lam M. H. Nuclear targeting signal recognition: a key control point in nuclear transport? Bioessays 22: 532 $544 ; 2000$

Kahle J.; Baake M.; Doenecke D.; Albig W. Subunits of the heterotrimeric transcription factor NF-Y are imported into the nucleus by distinct pathways involving importin beta and Imp13. Mol. Cell. Biol. 25(13): 5339-5354; 2005.

Kamei Y.; Yuba S.; Nakayama T.; Yoneda Y. Three distinct classes of the alpha-subunit of the nuclear pore-targeting complex (importin-alpha) are differentially expressed in adult mouse tissues. J. Histochem. Cytochem. 47(3): 363-372; 1999.

Kane J. K.; Hwang Y.; Konu O.; Loughlin S. E.; Leslie F. M.; Li M. D. Regulation of Homer and group I metabotropic glutamate receptors by nicotine. Eur. J. Neurosci. 21(5): 1145-1154; 2005.

Liang J.; Ke G.; You W.; Peng Z.; Lan J.; Kalesse M.; Tartakoff A. M.; Kaplan F.; Tao T. Interaction between Imp13 and myopodin suggests a nuclear import pathway for myopodin. Mol. Cell. Biochem. 307(1-2): 93-100; 2008.

Lin W.; Ye W.; Cai L.; Meng X.; Ke G.; Huang C.; Peng Z.; Yu Y.; Golden J. A.; Tartakoff A. M.; Tao T. The roles of multiple importins for nuclear import of murine aristaless-related homeobox protein. J. Biol. Chem. 284(30): 20428-20439; 2009.

Loveland K. L.; Hogarth C.; Szczepny A.; Prabhu S. M.; Jans D. A. Expression of nuclear transport importins beta 1 and beta 3 is regulated during rodent spermatogenesis. Biol. Reprod. 74(1): 67$74 ; 2006$

Macara I. G. Transport into and out of the nucleus. Microbiol. Mol. Biol. Rev. 65: 570-594; 2001.

Major A. T.; Whiley P. A.; Loveland K. L. Expression of nucleocytoplasmic transport machinery: clues to regulation of spermatogenic development. Biochim. Biophys. Acta 24: 1668-1688; 2011.

Mingot J. M.; Kostka S.; Kraft T.; Hartmann E.; Gorlich D. Imp13: a novel mediator of nuclear import and export. EMBO J. 20(14): 3685-3694; 2001

Nakamura T.; Arai Y.; Umehara H.; Masuhara M.; Kimura T.; Taniguchi H.; Sekimoto T.; Ikawa M.; Yoneda Y.; Okabe M.; Tanaka S.; Shiota K.; Nakano T. PGC7/Stella protects against DNA demethylation in early embryogenesis. Nat. Cell Biol. 9: 64-71; 2007.

Noctor S. C.; Flint A. C.; Weissman T. A.; Wong W. S.; Clinton B. K.; Kriegstein A. R. Dividing precursor cells of the embryonic cortical ventricular zone have morphological and molecular characteristics of radial glia. J. Neurosci. 22(8): 3161-3173; 2002.

Okada N.; Ishigami Y.; Suzuki T.; Kaneko A.; Yasui K.; Fukutomi R.; Isemura M. Importins and exportins in cellular differentiation. $J$. Cell. Mol. Med. 12: 1863-1871; 2008.

Perez-Terzic C.; Faustino R. S.; Boorsma B. J.; Arrell D. K.; Niederlander N. J.; Behfar A.; Terzic A. Stem cells transform into a cardiac phenotype with remodeling of the nuclear transport machinery. Nat. Clin. Pract. Cardiovasc. Med. 4(Suppl. 1): S68-S76; 2007.

Ploski J. E.; Shamsher M. K.; Radu A. Paired-typed homeodomain transcription factors are imported into the nucleus by karyopherin 13 Mol. Cell. Biol. 24(11): 4824-4834; 2004.

Quan Y.; Ji Z.; Wang X.; Tartakoff A. M.; Tao T. Evolutionary and transcriptional analysis of karyopherin beta superfamily proteins. Mol. Cell. Proteomics 7(7): 1254-1269; 2008.

Shoubridge C.; Cloosterman D.; Parkinson-Lawerence E.; Brooks D.; Gecz J. Molecular pathology of expanded polyalanine tract mutations in the Aristaless-related homeobox gene. Genomics 90: 59-71; 2007.

Su A. I.; Cooke M. P.; Ching K. A.; Hakak Y.; Walker J. R.; Wiltshire T.; Orth A. P.; Vega R. G.; Sapinoso L. M.; Moqrich A.; Patapoutian A.; Hampton G. M.; Schultz P. G.; Hogenesch J. B. Large-scale analysis of the human and mouse transcriptomes. Proc. Natl. Acad. Sci. U. S. A. 99: 4465-4470; 2002.

Tao T.; Lan J.; Presley J. F.; Sweezey N. B.; Kaplan F. Nucleocytoplasmic shuttling of $\operatorname{lgl} 2$ is developmentally regulated in fetal lung. Am. J. Respir. Cell Mol. Biol. 30(3): 350-359; 2004.

Tao T.; Lan J.; Lukacs G. L.; Hache R.; Kaplan F. Imp13 regulates nuclear import of the glucocorticoid receptor in airway epithelial cells. Am. J. Respir. Cell Mol. Biol. 35(6): 668-680; 2006.

Terry L. J.; Shows E. B.; Wente S. R. Crossing the nuclear envelope: hierarchical regulation of nucleocytoplasmic transport. Science 318: 1412-1416; 2007.

Tichopad A.; Dilger M.; Schwartz G.; Pfaffl M. W. Standardized determination of real-time PCR efficiency from a single reaction set-up. Nucl. Acids Res. 31(20): e122; 2003.

Wang H.; Tao T.; Tang J.; Mao Y. H.; Li W.; Peng J.; Tan G.; Zhou Y. P.; Zhong J. X.; Tseng S. C.; Kawakita T.; Zhao Y. X.; Liu Z. G. Imp13 serves as a potential marker for corneal epithelial progenitor cells. Stem Cells 27(10): 2516-2526; 2009.

Weis K. Nucleocytoplasmic transport: cargo trafficking across the border. Curr. Opin. Cell Biol. 14: 328-335; 2002. 
Whiley P. A.; Miyamoto Y.; McLachlan R. I.; Jans D. A.; Loveland K. L. Changing subcellular localization of nuclear transport factors during human spermatogenesis. Int. J. Androl. 35(2): 158-169; 2012.

Yamaguchi Y. L.; Tanaka S. S.; Yasuda K.; Matsui Y.; Tam P. P. Stagespecific Imp13 activity influences meiosis of germ cells in the mouse. Dev. Biol. 297(2): 350-360; 2006.

Yasuda Y.; Miyamoto Y.; Yamashiro T.; Asally M.; Masui A.; Wong C.; Loveland K. L.; Yoneda Y. Nuclear retention of importin $\alpha$ coordinates cell fate through changes in gene expression. $E M B O$ J. 31(1): 83-94; 2012.

Yasuhara N.; Shibazaki N.; Tanaka S.; Nagai M.; Kamikawa Y.; Oe S.; Asally M.; Kamachi Y.; Kondoh H.; Yoneda Y. Triggering neural differentiation of ES cells by subtype switching of importin-alpha. Nat. Cell Biol. 9: 72-79; 2007.

Yasuhara N.; Oka M.; Yoneda Y. The role of the nuclear transport system in cell differentiation. Semin. Cell Dev. Biol. 20: 590-599; 2009.

Young J. C.; Major A. T.; Miyamoto Y.; Loveland K. L.; Jans D. A. Distinct effects of importin $\alpha 2$ and $\alpha 4$ on Oct3/4 localization and expression in mouse embryonic stem cells. FASEB J. 25(11): 3958-3965; 2011.

Zhang C.; Sweezey N. B.; Gagnon S.; Muskat B.; Koehler D.; Post M.; Kaplan F. A novel karyopherin-beta homolog is developmentally and hormonally regulated in fetal lung. Am. J. Respir. Cell Mol. Biol. 22: 451-459; 2000. 